Document downloaded from:

http://hdl.handle.net/10251/161395

This paper must be cited as:

Arnal, J.; Chillarón-Pérez, M.; Parcero, E.; Súcar, LB.; Vidal-Gimeno, V. (2020). A Parallel Fuzzy Algorithm for Real-Time Medical Image Enhancement. International Journal of Fuzzy Systems. 22(8):2599-2612. https://doi.org/10.1007/s40815-020-00953-3

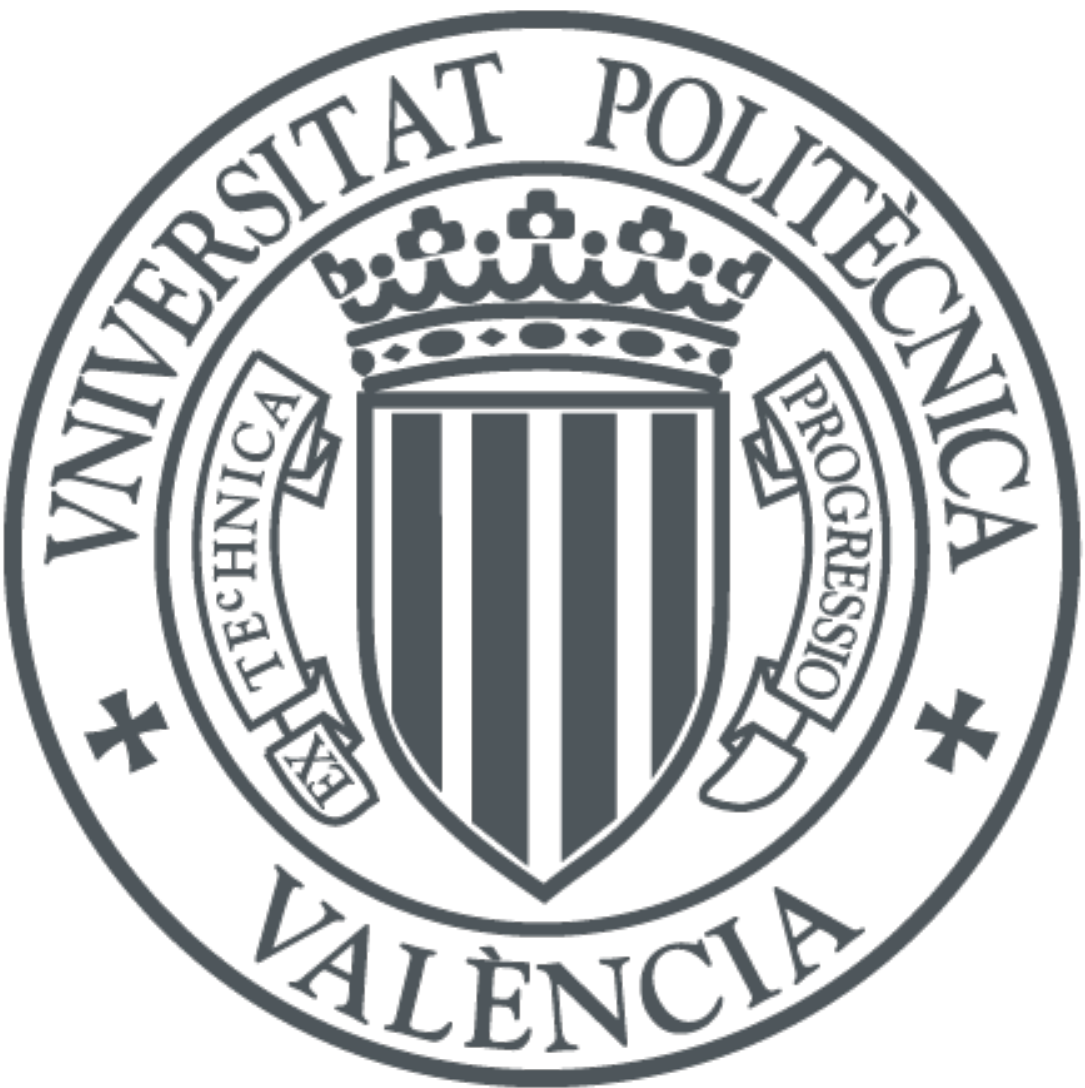

The final publication is available at

https://doi.org/10.1007/s40815-020-00953-3

Copyright Springer-Verlag

Additional Information 


\title{
A Parallel Fuzzy Algorithm for Real-Time Medical Image Enhancement
}

\author{
Josep Arnal • Mónica Chillarón • Estibaliz Parcero • Luis B. Súcar • \\ Vicente Vidal
}

Received: date / Accepted: date

\begin{abstract}
Medical images may be corrupted by noise. This noise affects the image quality and can obscure important information required for accurate diagnosis. Effectively apply filtering techniques can facilitate diagnosis or reduce radiation exposure. In this paper, we introduce a parallel method designed to reduce mixed Gaussian-impulse noise from digital images. The method uses fuzzy logic and the fuzzy peer group concept. Implementations of the method on multi-core interface using the Open Multi-Processing (OpenMP) and on graphics processing units (GPUs) using CUDA are presented. Efficiency is measured in terms of execution time and in terms of MAE, PSNR and SSIM over medi-
\end{abstract}

\author{
J. Arnal \\ Departamento de Ciencia de la Computación e Inteligencia \\ Artificial, Universidad de Alicante, San Vicente del Raspeig \\ 03690, Spain \\ Tel.: $+34965903400 \times 2985$ \\ Fax: +34 965909874 \\ E-mail: arnal@ua.es
}

M. Chillarón

Departamento de Sistemas Informáticos y Computación, Universidad Politécnica de Valencia, Valencia 46730, Spain

\section{E. Parcero}

Instituto de Tecnologías de la Información y Comunicaciones, Universidad Politécnica de Valencia, Valencia 46022, Spain

\section{L.B. Súcar}

Departamento de Ciencia de la Computación e Inteligencia Artificial, Universidad de Alicante, San Vicente del Raspeig 03690, Spain

\section{Vidal}

Departamento de Sistemas Informáticos y Computación, Universidad Politécnica de Valencia, Valencia 46730, Spain cal images from the mini-MIAS database and over Computed Radiography (CR) images generated at different exposure levels. These images have been contaminated with impulsive and/or Gaussian noise. Experiments show that the proposed method obtains good performance in terms of the above mentioned objective quality measures. After applying multi-core and GPUs optimization strategies, the observed time shows that the new filter allows to remove mixed Gaussian-impulse noise in real-time.

Keywords Filter design - Medical image processing . Fuzzy logic $\cdot$ Noise reduction

\section{Introduction}

Filtering methods, i.e. techniques to detect and reduce noises, are essential in medical imaging (e.g., X-Rays, Magnetic Resonance Imaging (MRI), Computer Tomography $(\mathrm{CT})$ ) because the quality of the image can have repercussions on the diagnosis of a disease (for example, detecting microcalcifications in a mammogram). Moreover, noise reduction filters can be used to improve images when a reduced radiation dose is used $[14,15]$. This fact is especially crucial in CT images in order to reduce the exposure to X-Rays because the amount of radiation tends to be very high. Two specially common types of noise are the impulsive noise and the Gaussian noise. The impulsive noise is introduced during the transmission process and the Gaussian during the the acquisition process $[2,27]$. A large number of algorithms have been introduced to reduce either Gaussian (see e.g. $[9,10,19,27,29,38]$ ) or impulse noise (see e.g. $[3-5,21,23,30-34,39])$. However, not all methods are useful when images are contaminated simultaneously with impulsive and Gaussian noise. A possible approach 
to address this problem is to perform consecutively two filters to remove first Gaussian noise and then the impulses, or vice versa. But, the use of two consecutive methods could significantly reduce the computational performance and therefore this approach could not be feasible for real cases.

In [22] authors proposed a color image filter for the removal of mixed Gaussian-impulse noise. This method, named Fuzzy Peer Group Averaging Filter (FPGA), used the fuzzy peer group concept. Experimental results showed that this filtering technique exhibits competitive results when processing color images compared to other state-of-the-art methods but has not been tested in the field of medical images. On the other hand, due to the large size of high-resolution images, sequential computers are not able to perform this algorithm in real-time. Then, FPGA filter has demonstrated to obtain satisfactory results in quality but is not applicable for real-time processing. Nowadays parallel computing is one of the most appropriate ways to obtain real-time results or to reduce the computational time in all kind of applications [7,43-45]. Moreover, due to the advances in cloud computing technology $[18,20]$ it has become possible to run parallel algorithms without much inhouse resources [42].

Due to these causes, in this work we introduce a new parallel algorithm based on filters introduced in [22] with the purpose of improving their computational efficiency, so as to make them appropriate for real-time processing.

We have analyzed this parallel algorithm programming codes for multi-cores and GPUs, obtaining good speedup results. A nearly linear speedup with the number of processors used has been achieved for multi-cores. Nowadays, multi-cores and GPUs are widely available, and then the introduced approach is a practical, and efficient technique for real-time image processing. In the experiments, we used the mammograms obtained form the mini-MIAS database [37] and Computed Radiography (CR) images generated at different exposure levels. The filter performance has been evaluated using the Mean Absolute Error (MAE), Peak Signal to Noise Ratio (PSNR), and the Structural Similarity In$\operatorname{dex}(\mathrm{SSIM})[27,41]$.

This work is organized into four sections. Section 2 explains the proposed parallel noise reduction algorithm, experiments are shown in Section 3, and the conclusions are presented in Section 4.

\section{Parallel Noise Reduction Method}

Let consider the color image $A$ defined as a mapping $\mathbb{Z}^{2} \rightarrow \mathbb{Z}^{3}$. That is, the image is given by a matrix $A$ of

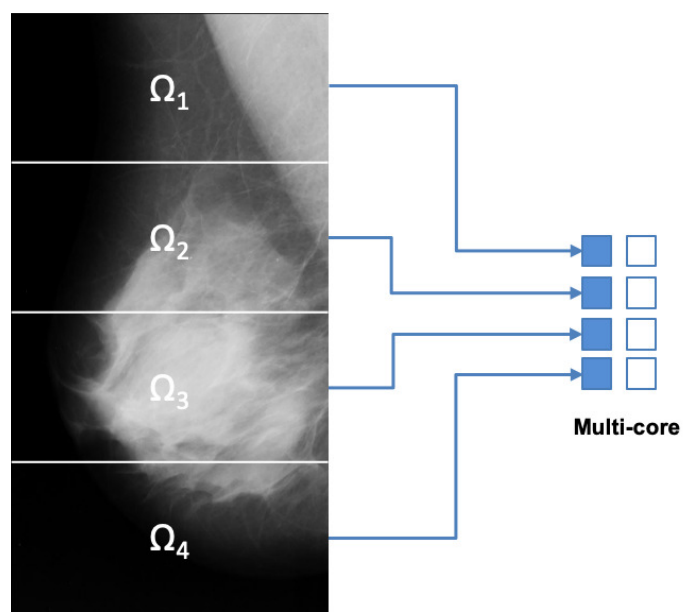

Fig. 1 Image domain decomposition: Distributed image on 4 cores

size $M \times N$ consisting of pixels $x_{i}=\left(x_{i}(1), x_{i}(2), x_{i}(3)\right)$, indexed by $i$, that gives the position of the pixel on the image domain $\Omega$. The vector components $x_{i}(\ell)$, for $i=$ $1,2, \ldots, M \times N$ and $\ell=1,2,3$, which are quantified into the integer domain, represent the RGB color channel values.

Let $W$ be a filtering window of $n \times n$ pixels centered at pixel $x_{0}$. And let $x_{i} \in W, i=1, \ldots, n^{2}-1$ represent the neighbor pixels of $x_{0}$. The parallel method proposed in this paper uses the fuzzy peer group concept according to [22] and uses a fuzzy metric. With the purpose of describing the parallel method, we split the image domain $\Omega$ into $P$ subdomains $\left\{\Omega_{i}\right\}_{i=1}^{P}$, being $P$ the number of computation elements. This domain decomposition satisfies

$$
\Omega_{i} \subset \Omega, \bigcup_{i=1,2, \ldots, P} \Omega_{i}=\Omega \text {, and } \Omega_{i} \cap \Omega_{j}=\emptyset \text { for } i \neq j \text {. }
$$

Fig. 1 presents an example of the decomposition employed in the numerical experiments. In this case, the image is distributed into four subdomains. In order to process pixels in the inner border of the subdomains, each computing element needs some additional pixels. Then we consider an overlapping domain decomposition of the image. Fig. 2 illustrates an overlapping and a non-overlapping domain decomposition for four subdomains. For the overlapping domain decomposition, we define $\Omega_{i}^{w}, i=1, \ldots, P$; an extension of $\Omega_{i}$ where $\omega$ is a non-negative integer determining the size of the overlapping region. Computing element $i$ processes pixels in domain $\Omega_{i}$, but using pixels in domain $\Omega_{i}^{w} . \Omega$ is equal to the integer part of $n / 2$, where $n \times n$ is the size of the filtering window. Then, in order to remove the impulses a fuzzy rule based scheme is used. This procedure employes the fuzzy peer group definition. In order 


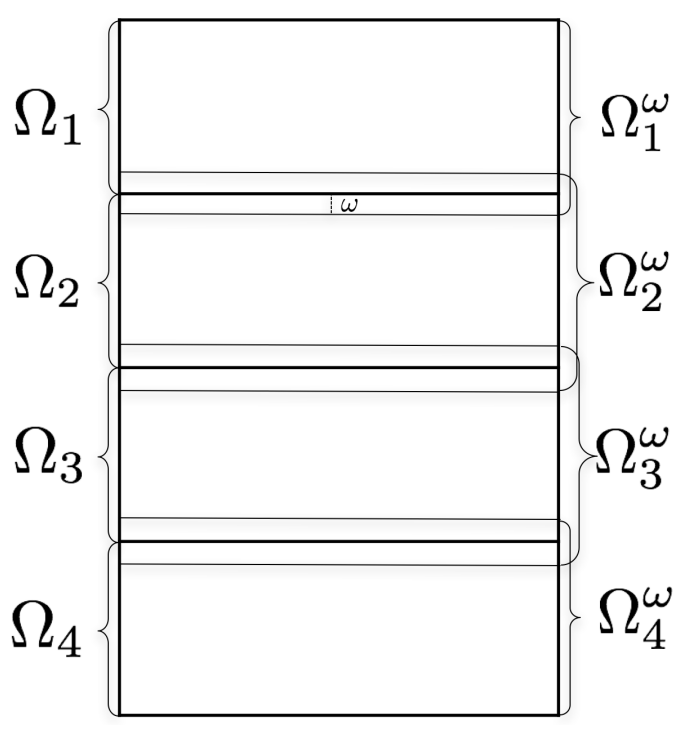

Fig. 2 Overlapping domain decomposition for four subdomains

to reduce Gaussian noise, we performed a fuzzy averaging among the pixels of the fuzzy peer group. Algorithm 1 and Fig. 3 show the parallel filtering algorithm and the corresponding flowchart. The following paragraphs explain the steps of the algorithm.

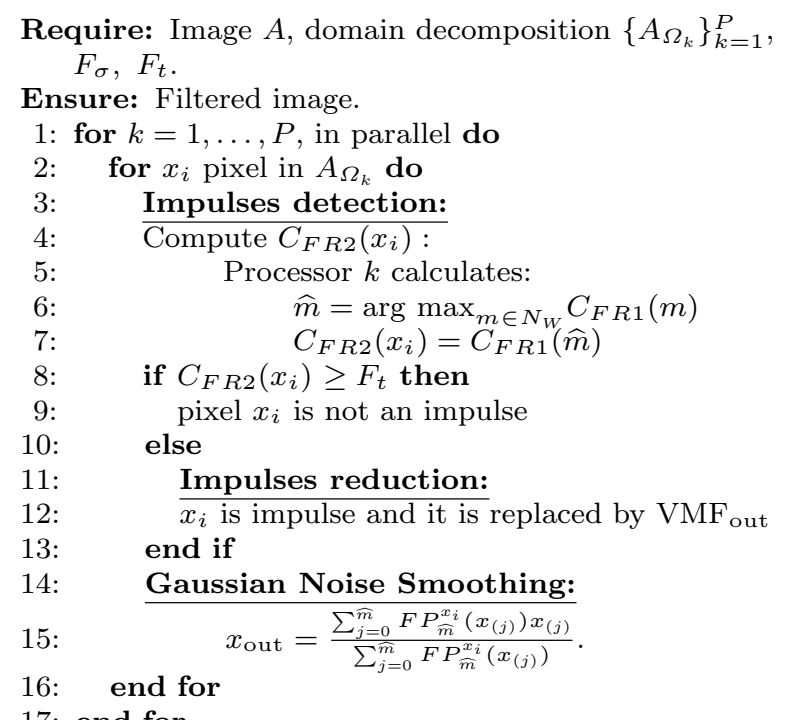

\section{7: end for}

Algorithm 1: Parallel denoising method.

The concept of peer group [17] is based on the ordering of the neighbor pixels considering its similarity to the central pixel $x_{0}$. Let $S$ be a convenient similarity measure [27] between two pixels. Pixels $x_{i} \in W$ are ordered in a descending order based on their similarity to $x_{0}$. Then, an ordered set $W^{\prime}=\left\{x_{(0)}, x_{(1)}, \ldots, x_{\left(n^{2}-1\right)}\right\}$ is obtained, such that $S\left(x_{0}, x_{(0)}\right) \geq S\left(x_{0}, x_{(1)}\right) \geq \cdots \geq$

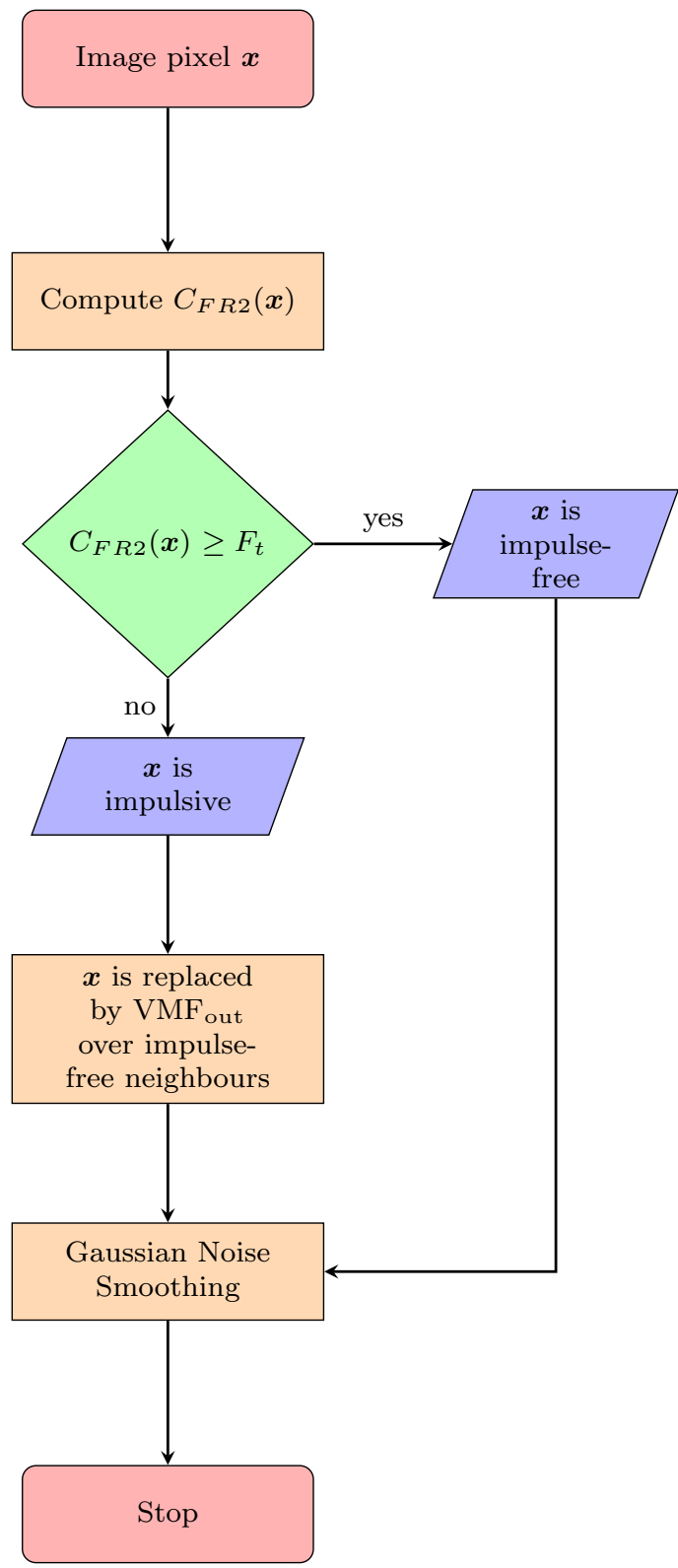

Fig. 3 Flowchart describing the filtering algorithm.

$S\left(x_{0}, x_{\left(n^{2}-1\right)}\right)$, where $x_{(0)}=x_{0}$. Then, according to the concept of peer group [17], the peer group $\mathcal{P}\left(m, x_{0}\right)$ of $m+1$ pixels associated with $x_{0}$ is given by the set

$\mathcal{P}\left(m, x_{0}\right)=\left\{x_{(0)}, x_{(1)}, \ldots, x_{(m)}\right\}$.

In [22], a fuzzy logic algorithm is presented to compute the best number of pixels $\widehat{m}$ in a peer group. The fuzzy peer group for the central pixel $x_{0}$ in a window $W$ according to $[22]$ is given by the fuzzy set $\mathcal{F} \mathcal{P}\left(\widehat{m}, x_{0}\right)$ determined on the set $\left\{x_{(0)}, x_{(1)}, \ldots, x_{(\widehat{m})}\right\}$ and defined by the membership function $F P_{\widehat{m}}^{x_{0}}=S\left(x_{0}, x_{(i)}\right)$.

Then, the best number $\widehat{m}$ of pixels of $\mathcal{P}\left(m, x_{0}\right)$ is determined as the number $m \in \mathcal{N}_{W}=\left\{1,2, \ldots, n^{2}-1\right\}$ which maximizes the certainty of the Fuzzy Rule FR1. 


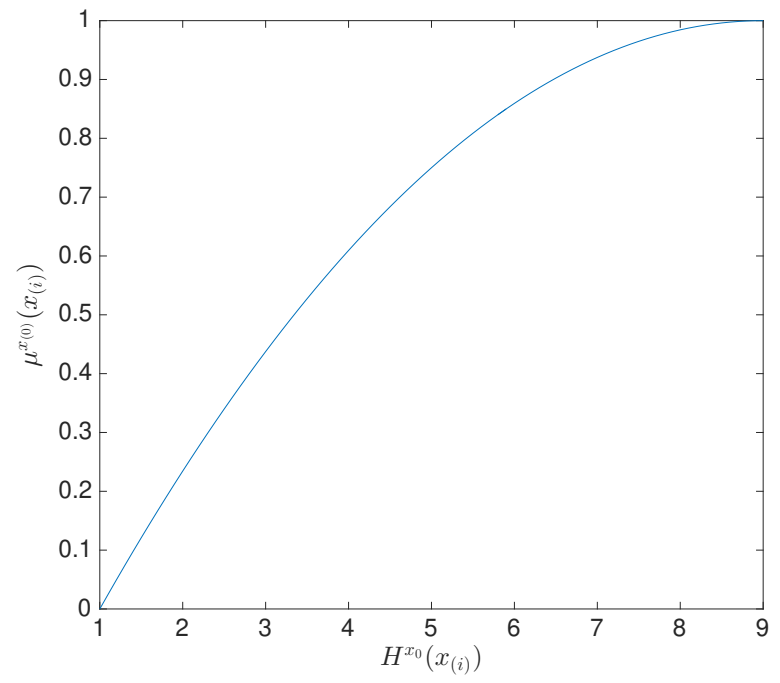

Fig. 4 Membership function (4) used to compute the certainty of $H^{x_{0}}\left(x_{(i)}\right)$ is large

Fuzzy Rule FR1: Certainty for $m$ to be the best number of pixels in $\mathcal{P}\left(m, x_{0}\right)$

IF " $x_{m}$ is similar to $x_{0}$ " and the accumulated similarity for $x_{(m)}$ is large

THEN "the certainty for $m$ to be the best number of pixels for $\mathcal{P}\left(m, x_{0}\right)$ is high".

$C_{F R 1}(m)$ represents the certainty of the Fuzzy Rule FR1 for $m$. Then, $C_{F R 1}(m)$ is calculated for each number $m \in \mathcal{N}_{W}$ and the $m$ maximizing the certainty is selected as the best number $\widehat{m}$ of pixels in $\mathcal{P}\left(m, x_{0}\right)$, i.e., $\widehat{m}=\arg \max _{m \in N_{W}} C_{F R 1}(m)$.

The certainty of " $x_{m}$ is similar to $x_{0}$ " is determined by the membership function $C^{x_{0}}$ given by the similarity function

$C^{x_{0}}\left(x_{(i)}\right)=S\left(x_{0}, x_{(i)}\right), i,=0,1 \ldots, n^{2}-1$.

The accumulated similarity for $x_{(m)}$, represented by $H^{x_{0}}\left(x_{(m)}\right)$, is given by

$H^{x_{0}}\left(x_{(i)}\right)=\sum_{k=0}^{k=i} S\left(x_{0}, x_{(k)}\right), i \in\left\{0,1, \ldots, n^{2}-1\right\}$.

Then, the certainty of " $H^{x_{0}}\left(x_{(m)}\right)$ is large" is determined by the membership function $\mu^{x_{0}}$ (see Fig. 4) defined by

$\mu^{x_{0}}\left(x_{(i)}\right)=-\frac{\left(H^{x_{0}}\left(x_{(i)}\right)-1\right)\left(H^{x_{0}}\left(x_{(i)}\right)-2 n^{2}+1\right)}{\left(n^{2}-1\right)^{2}}$
$i=0,1, \ldots, n^{2}-1$.

As conjunction operator, the product t-norm was used, and therefore no defuzzification was needed. Therefore, $C_{F R 1}(m)=C^{x_{0}}\left(x_{(m)}\right) \mu^{x_{(0)}}\left(x_{(m)}\right)$.
The fuzzy similarity function employed was

$S\left(x_{i}, x_{j}\right)=e^{-\frac{\left\|x_{i}-x_{j}\right\|}{F_{\sigma}}} \quad i, j=0, \ldots, n^{2}-1$

where $\|\cdot\|$ is the Euclidean norm and $F_{\sigma}$ denotes a parameter that will be analyzed in Section 3. This function has been chosen because it is a fuzzy metric $[12,13]$, and this type of fuzzy metrics has been demonstrated to be convenient for fuzzy image processing [3, 22-24]. The similarity $S$ is valued in the interval [0,1], and $S\left(x_{0}, x_{i}\right)=1$ if and only if $x_{0}=x_{i}$.

Moreover, a fuzzy rule is employed to detect impulses (step 3 in Algorithm 1).

Fuzzy Rule FR2: Certainty for pixel $x_{0}$ not to be an impulse

IF " $H^{x_{0}}\left(x_{(\widehat{m})}\right)$ is large" and " $x_{(\widehat{m})}$ is similar to $x_{0}$ " THEN " $x_{0}$ is not an impulse".

With the purpose of calculating the certainty of Fuzzy Rule FR2, represented by $C_{F R 2}$, the certainty of " $H^{x_{0}}\left(x_{(\widehat{m})}\right)$ is large" is given by $\mu^{x_{(0)}}(4)$, and the certainty of " $x_{(\widehat{m})}$ is similar to $x_{0}$ " is determined by $C^{x_{0}}$ (2). As conjunction operator, the t-norm product is employed, and therefore $C_{F R 2}\left(x_{0}\right)=C^{x_{0}}\left(x_{(\widehat{m})}\right) L^{x_{0}}\left(x_{(\widehat{m})}\right)$. This certainty is already computed since $C_{F R 2}\left(x_{0}\right)=$ $C_{F R 1}(\widehat{m})$, and therefore, it is not needed additional computation. If $C_{F R 2}$ satisfies

$C_{F R 2}\left(x_{(0)}\right) \geq F_{t}$

then $x_{0}$ is not an impulse, else $x_{0}$ is corrupted with impulsive noise and it is substituted by $\mathrm{VMF}_{\text {out }}[1] . F_{t}$ denotes a threshold parameter valued in the interval $[0,1]$ which will be analyzed in Section 3 .

\section{Experimental Results}

We have coded two parallel implementations. One on multi-core with OpenMP $[11,25]$ and the second using CUDA [8] on GPUs. OpenMP is an application programming interface (API) that supports multi-platform shared memory parallel programming in languages Fortran, $\mathrm{C}$ or $\mathrm{C}++$, on most operating systems. CUDA is an API that allows to use a CUDA-enabled GPU for parallel programming. CUDA works with programming languages such as Fortran, $\mathrm{C}$ or $\mathrm{C}++$, and moreover supports programming frameworks such as OpenACC and OpenCL. We developed experiments using three different machines:

- Multi-core: Intel Xeon CPU E5320 (8 cores), 1.86 GHz, 42 GB RAM, Linux Ubuntu 16.04.4.

- GPU 1: NVIDIA Tesla K20 GPU with 5 GB of GDDR5 on-board memory, 2496 processor cores, $706 \mathrm{MHz}$ processor core clock and $208 \mathrm{~GB} / \mathrm{sec}$ memory bandwidth. 
- GPU 2: NVIDIA Tesla V100 GPU with 32 GB of HBM2 on-board memory, 5120 processor cores, 1230 $\mathrm{MHz}$ processor core clock and $897 \mathrm{~GB} / \mathrm{sec}$ memory bandwidth.

Two different sets of images were used in the experiments:

- Images from the mini-MIAS database,

- Computed Radiography (CR) images generated at different exposure levels.

Different real mammograms obtained from the miniMIAS database [37] were used in the experiments (see Fig. 5). Moreover, a detail of each mammogram was used in order to better perceive the performance of the filtering technique (see Fig. 6). mdb001 corresponds to a fatty-glandular breast with benign abnormalities and well-defined/ circumscribed masses. mdb005 is a fatty breast with benign abnormalities and well-defined/ circumscribed masses. mdb006 corresponds to a fatty normal breast and mdb248 is a fatty breast with benign abnormalities and calcifications. Moreover, a chest of a female Alderson RANDO ${ }^{\circledR}$ was used. This phantom consists in a synthetic human skeleton inside a mass comparable to human soft tissue. This phantom is designed to have levels of absorption similar to human tissue exposed to a radiation dose. The phantom was scanned with an AGFA imaging device at 1, 0.8, 0.6, $0.4 \mathrm{mAs}$ using 70 and 80 kilovoltage $(\mathrm{kV})$. Fig. 7 show the phantom, and an example of a CR image obtained at $80 \mathrm{kV}$ and $0.4 \mathrm{mAs}$. A detail of the CR images, with size of $501 \times 501$ pixels, was used in the experiments.

These images have been contaminated with impulsive and/or Gaussian noise. The classical white additive Gaussian noise [27] has been used, and random-value impulse noise [27] was considered. In order to evaluate the filter efficiency we have used the commonly employed objective measures MAE, PSNR and the SSIM. The MAE, and PSNR are defined as follows [27]:

$$
\begin{aligned}
\mathrm{MAE} & =\frac{1}{Q M N} \sum_{k=1}^{Q} \sum_{i=1}^{M N}\left|o_{i k}-x_{i k}\right|, \\
\mathrm{PSNR} & =20 \log \left(\frac{255}{\sqrt{\frac{1}{Q M N} \sum_{k=1}^{Q} \sum_{i=1}^{M N}\left(o_{i k}-x_{i k}\right)^{2}}}\right),
\end{aligned}
$$

where $M, N$ are te image dimensions, $Q$ is the number of channels of the image, $o_{i k}$ is the component $k$ of the original pixel $o_{i}, x_{i k}$ is the component $k$ of the noisy or filtered pixel $x_{i}$ and $i$ is the pixel position in the image. The SSIM is defined as [41]:

$\operatorname{SSIM}(x, y)=\frac{\left(2 \mu_{x} \mu_{y}+c_{1}\right)\left(2 \sigma_{x y}+c_{2}\right)}{\left(\mu_{x}^{2}+\mu_{y}^{2}+c_{1}\right)\left(\sigma_{x}^{2}+\sigma_{y}^{2}+c_{2}\right)}$ where $\mu_{x}, \mu_{y}, \sigma_{x}, \sigma_{y}, \sigma_{x y}$ are the local means, standard deviations, and covariance for images $x, y$, and $c_{1}, c_{2}$ are two constants to stabilize the division with weak denominator. MAE is used to evaluate detail preservation, PSNR are used to evaluate noise reduction capability and the SSIM measures the similarity between two images. For an efficient filter, it is expected to obtain high PSNR and SSIM (the limit for SSIM is 1), and low values for the other parameter MAE.

In order to tune in the parameters $F_{\sigma}$ in $(5)$ and $F_{t}$ in (6), the algorithm performance has been evaluated in terms of PSNR as a function of $F_{t}$ and $F_{\sigma}$, corrupting images with different intensities of Gaussian and impulsive noise. The best results for $F_{t}$ were achieved taking $F_{t}=0.85$ when $\left.\left.p \in\right] 0.05,0.1\right]$ and $F_{t}=0.15$ when $p \in] 0,0.05]$. For $F_{\sigma}$ the best results were obtained for $F_{\sigma}=480$ when $\left.\left.\sigma \in\right] 5,10\right]$ and $F_{\sigma}=100$ when $\left.\left.\sigma \in\right] 0,5\right]$.

We compare the new method with other outstanding filters which have been applied satisfactorily in medical images: Non-linear Difussive Filter (NDF) [16], the Fuzzy Peer Group Fuzzy Metric Filter (FPG) [3] and the Peer Group-Fuzzy Non-linear Diffusion Filter (FPGNDF). The NDF filter belongs to a competitive class of filters based on variational and partial differential equations, such as the anisotropic diffusion [26], total variation [28], and related works [16,40]. The FPG filter is based on the peer group technique and fuzzy metric $[3,35]$. The FPG-NDF filter is the combination of NDF and FPG. In addition, the new filter has been compared with two other outstanding methods specifically designed to reduce mixed Gaussian-impulsive noise: SFMR filter [6] and RLSF filter [36]. These filters have been implemented setting the optimal parameters determined by the corresponding authors.

Tables 1, 2 and 3 show, respectively, the MAE, PSNR and SSIM performance for different intensities of Gaussian and impulse noise including images corrupted only with Gaussian noise (see Tables 1, 2 and 3 with $p=0$ ) and only with impulse noise (Tables 1,2 and 3 with $\sigma=$ $0)$. These results show that the new method presents the best behavior in almost all experiments in terms of the MAE, PSNR, and the SSIM measures. Only in the case of images exclusively corrupted with impulsive noise, the FPG filter specifically designed for impulses, achieve a small advantage in terms of MAE. This implies that the new method presents a better noise reduction and a better image details preservation. Values of these objective mesures show that the method obtains a robust performance. Moreover, these results support the effectiveness of the algorithm even to process images contaminated only with Gaussian noise or only impulsive noise. The same behavior can be ob- 

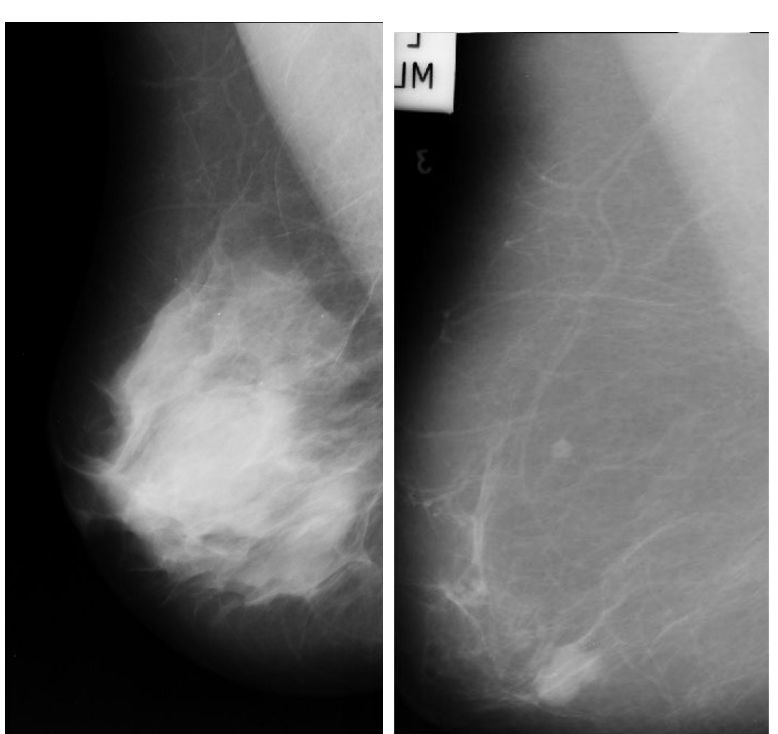

(a) mdb001 $512 \times 969$ pixels (b) mdb005 $512 \times 960$ pixels (c) mdb006 $512 \times 960$ pixels

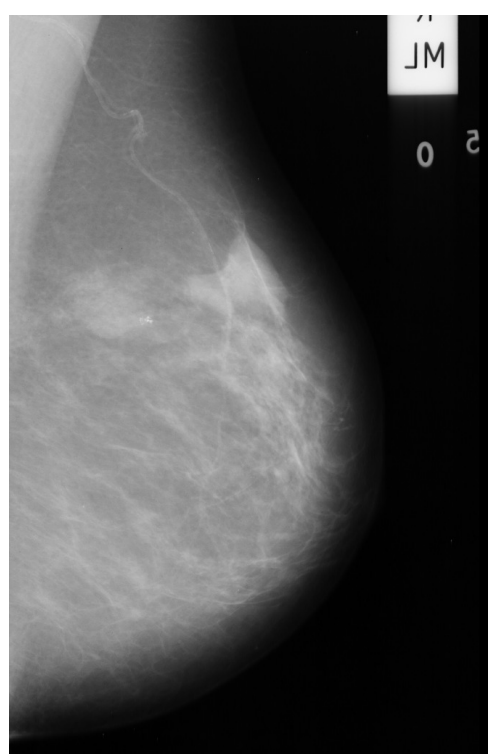

(d) mdb248 $660 \times 1024$ pixels

Fig. 5 Test mammograms from mini-MIAS database [37]

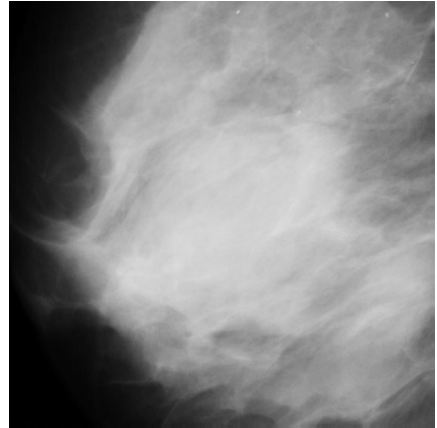

(a) mdb001 $400 \times 400$ pixels

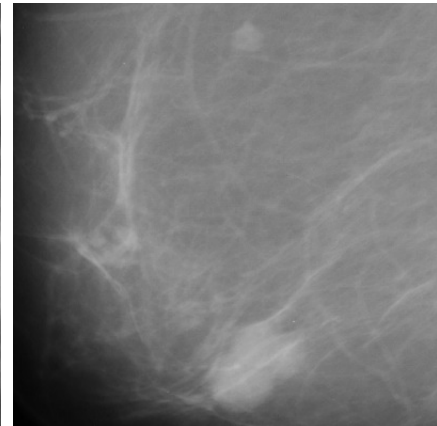

(b) mdb005 $400 \times 400$ pixels

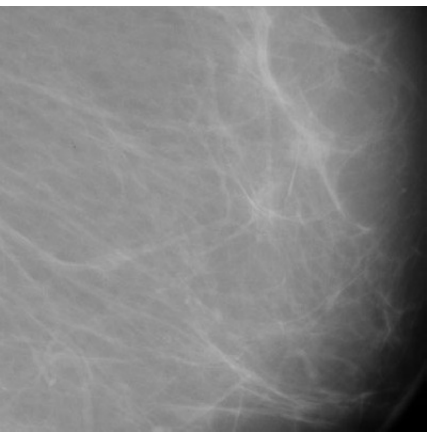

(c) mdb006 $400 \times 400$ pixels

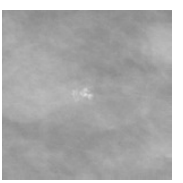

(d) $\operatorname{mdb} 248$ $150 \times 150$ pixels

Fig. 6 Details from test mammograms

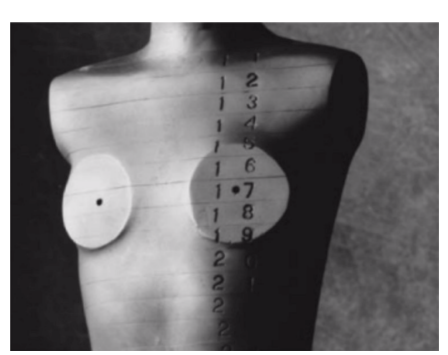

(a)

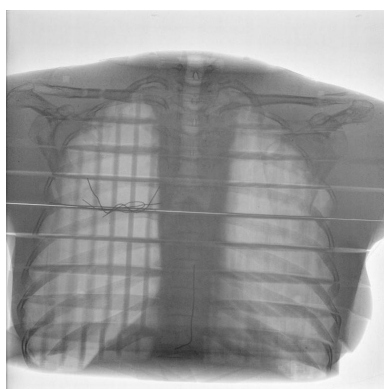

(b)

Fig. 7 (a) Female Alderson RANDO phantom; (b) CR $(3730 \times 3062$ pixels $)$ from RANDO phantom at $70 \mathrm{kV}$ and $1 \mathrm{mAs}$. 
served (see Tables 4, 5 and 6 ) for CR images generated at different exposure levels.

From the visual point of view, by inspecting the denoised images in Fig. 8, it can be concluded that the new filter obtains robust results improving the NDF, FPG, FPG-NDF, RLSF and SFRF filters. It can be observed that the RLSF method blurs image edges more than the new filter, missing edges and small details. The SFRF method does not eliminate impulses as effectively as the proposed method. Figs. 9, 10, and 11 show that the new technique efficiently detect and reduce impulsive noise as well as effectively smooth Gaussian noise while preserving the quality of details and edges, being able to better appreciate the breast abnormalities. Even small details are preserved as the microcalcifications in Fig. 11. The same behavior can be seen in the CR images for the different exposure levels (Figs. 12 and 13). We designed both the serial code and parallel code and then compared the execution time. Fig. 1 presents an example of the image decomposition employed in the experiments using 4 cores. In the implementation on GPU the kernel was configured in three phases illustrated in Fig. 14. Each phase cannot start until the previous phase is terminated. To assure this synchronization, the threads are synchronized after the impulsive noise detection phase and after the impulsive noise removing. On the GPU, the kernel was designed so that each thread processed one pixel.

To quantify parallel performance, parallel speedup $S_{P}$ is computed as:

$S_{P}=\frac{T_{s e q}}{T_{P}}$

where $T_{\text {seq }}$ is the computational time of the sequential algorithm and $T_{P}$ is the computational time of the parallel method. We compare the parallel algorithm on Multi-core and GPU with the sequential one running on Multi-core.

Table 7 presents the computational times for the mammogram mdb006 $(512 \times 960$ pixels $)$ and Table 8 presents the computational time for the CR image (3730 $\times 3062$ pixels) from RANDO phantom at $70 \mathrm{kV}$ and $1 \mathrm{mAs}$ (Fig. 7). The results show that a significant speedup is achieved. In particular, the global efficiencies achieved with respect to the sequential method were on Multi-core about $93-100 \%$ for $P=2,92-96 \%$ for $P=4,88-96 \%$ for $P=6$ and $69-89 \%$ for $P=8$, depending on the image size. In the GPU 1 a large speedup of 1000 units can been obtained. In the GPU 2, more powerful than GPU 1, a speedup exceeding 8300 units can be achieved. Similar results were achieved for the other images. The times observed in Tables 7 and 8 show that the new method allows the processing of large medical images in real time.
3.1 Application to the reduction of the radiation dose in $\mathrm{CR}$ images

A methodology used to reduce the dose to patients in $\mathrm{X}$-ray computed radiography, consists in decreasing the exposure (mAs) and then, filter the image with an appropriate filter. In order to analyze the similarity with a CR image of $1 \mathrm{mAs}$, we compute an estimation of the standard deviation (SD) of the output of the new filter. For this purpose we consider a detail of the CR images with size $501 \times 501$. Table 9 presents the SD values for the $\mathrm{CR}$ images at $70 \mathrm{kV}$. It can be observed that the $\mathrm{SD}$ values of the new filter are lower than those corresponding to the filters PFG-NDF, RLSF and SFRF for all the cases. Moreover, these values are also lower than the SD value of the original $\mathrm{CR}$ image at $1 \mathrm{mAs}$ (18.4855). This fact means that the images generated by the new filter correspond to $\mathrm{CR}$ images with a higher radiation dose than $1 \mathrm{mAs}$. Table 9 shows the same behavior for the CR images generated at $80 \mathrm{kV}$. Using this property the new filter can be used to reduce the radiation dose.

\section{Conclusion}

A parallel method based on fuzzy peer groups and fuzzy logic has been presented to detect and reduce mixed Gaussian-impulsive noise in medical images. The method has been implemented on multi-cores using OpenMP and GPUs using CUDA. The implementations have been used to reduce the impulsive noise, Gaussian and a mix of both of them on mammograms from the miniMIAS database and on CR images generated at different exposure levels. The filter obtained robust results in terms of the objective quality mesures MAE, PSNR and SSIM. The parallel algorithm introduced demonstrated a significant speedup, resulting in reduced computational times that make the new method appropriate for real-time medical image processing. The method minimizes the standard deviation for a CR image obtained with low radiation exposure (mAs). In future works, we will analyze the use of this technique to reduce other types of noise present in medical images from CT, MRI, US and PET.

Acknowledgements This research was supported by the Spanish Ministry of Science, Innovation and Universities (Grant RTI2018-098156-B-C54) co-financed by FEDER funds.

\section{References}

1. Astola, J., Haavisto, P., Neuvo, Y.: Vector median filters. Proc. IEEE 78(4), 678-689 (1990) 
Table 1 MAE for noisy and filtered images. Mammogram mdb006

\begin{tabular}{ll|ccccccc}
\hline \multirow{2}{*}{ Noise } & \multicolumn{5}{c}{ MAE } \\
Gaussian & Impulsive & Noisy & FPG & NDF & FPG-NDF & RLSF & SFRF & New filter \\
\hline$\sigma=10$ & $p=0$ & 19.67 & 13.26 & 7.32 & 6.98 & 6.74 & 6.53 & 6.47 \\
$\sigma=0$ & $p=0.1$ & 12.82 & 0.17 & 9.48 & 0.75 & 1.95 & 1.72 & 1.12 \\
$\sigma=10$ & $p=0.1$ & 29.66 & 13.66 & 12.56 & 7.34 & 7.01 & 6.95 & 6.61 \\
\hline
\end{tabular}

Table 2 PSNR for noisy and filtered images. Mammogram mdb006

\begin{tabular}{ll|llrrrrr}
\hline \multicolumn{2}{c}{ Noise } & \multicolumn{9}{c}{ PSNR } \\
Gaussian & Impulsive & Noisy & FPG & NDF & FPG-NDF & RLSF & SFRF & New filter \\
\hline \multirow{2}{*}{$\sigma=10$} & $p=0$ & 17.07 & 23.27 & 28.66 & 28.14 & 28.50 & 29.21 & 30.12 \\
$\sigma=0$ & $p=0.1$ & 15.33 & 40.69 & 24.39 & 39.56 & 40.32 & 41.43 & 43.99 \\
$\sigma=10$ & $p=0.1$ & 13.73 & 23.03 & 23.36 & 27.99 & 28.23 & 28.41 & 29.38 \\
\hline
\end{tabular}

Table 3 SSIM for noisy and filtered images. Mammogram mdb006

\begin{tabular}{ll|ccccccc}
\hline \multicolumn{2}{c}{ Noise } & \multicolumn{9}{c}{ SSIM } \\
Gaussian & Impulsive & Noisy & FPG & NDF & FPG-NDF & RLSF & SFRF & New filter \\
\hline$\sigma=10$ & $p=0$ & 0.13 & 0.24 & 0.76 & 0.79 & 0.81 & 0.83 & 0.87 \\
$\sigma=0$ & $p=0.1$ & 0.10 & 0.88 & 0.31 & 0.90 & 0.91 & 0.93 & 0.97 \\
$\sigma=10$ & $p=0.1$ & 0.08 & 0.31 & 0.37 & 0.70 & 0.72 & 0.75 & 0.82 \\
\hline
\end{tabular}

Table 4 MAE for noisy and filtered images. CR images generated at different exposure levels.

\begin{tabular}{ll|ccccccc}
\hline \multicolumn{2}{c}{ Noise } & \multicolumn{7}{c}{ MAE } \\
\hline Gaussian & Impulsive & Noisy & FPG & NDF & FPG-NDF & RLSF & SFRF & New filter \\
\hline CR images at $70 \mathrm{kV}$ with $0.4 \mathrm{mAs}$ \\
\hline$\sigma=10$ & $p=0$ & 19.96 & 14.16 & 7.98 & 6.32 & 5.89 & 5.43 & 5.35 \\
$\sigma=0$ & $p=0.1$ & 13.02 & 0.16 & 8.22 & 0.71 & 1.78 & 1.53 & 0.81 \\
$\sigma=10$ & $p=0.1$ & 30.21 & 14.45 & 13.34 & 6.54 & 6.12 & 5.94 & 5.52 \\
\hline CR images at $80 \mathrm{kV}$ with $1 \mathrm{mAs}$ & & & & & \\
\hline$\sigma=10$ & $p=0$ & 18.34 & 13.89 & 7.87 & 6.18 & 5.23 & 5.06 & 4.21 \\
$\sigma=0$ & $p=0.1$ & 12.92 & 0.15 & 7.98 & 0.70 & 1.56 & 1.48 & 0.72 \\
$\sigma=10$ & $p=0.1$ & 28.23 & 10.19 & 9.24 & 5.18 & 4.85 & 4.62 & 4.35 \\
\hline
\end{tabular}

Table 5 PSNR for noisy and filtered images. CR images generated at different exposure levels.

\begin{tabular}{ll|lllllll}
\hline \multicolumn{2}{c}{ Noise } & \multicolumn{7}{c}{ PSNR } \\
\hline Gaussian & Impulsive & Noisy & FPG & NDF & FPG-NDF & RLSF & SFRF & New filter \\
\hline \multicolumn{2}{l}{ CR images at $70 \mathrm{kV}$ with $0.4 \mathrm{mAs}$} \\
\hline$\sigma=10$ & $p=0$ & 17.60 & 22.89 & 28.40 & 28.23 & 28.34 & 29.13 & 30.32 \\
$\sigma=0$ & $p=0.1$ & 16.52 & 41.45 & 26.86 & 39.87 & 40.67 & 41.23 & 42.78 \\
$\sigma=10$ & $p=0.1$ & 14.93 & 23.76 & 25.32 & 29.17 & 29.39 & 29.60 & 30.66 \\
\hline \multicolumn{7}{l}{ CR images at $80 \mathrm{kV}$ with $1 \mathrm{mAs}$} \\
\hline$\sigma=10$ & $p=0$ & 17.31 & 24.56 & 29.01 & 28.98 & 29.56 & 30.26 & 31.98 \\
$\sigma=0$ & $p=0.1$ & 16.45 & 42.12 & 25.35 & 41.23 & 42.23 & 43.21 & 45.35 \\
$\sigma=10$ & $p=0.1$ & 14.38 & 24.51 & 28.93 & 29.51 & 29.94 & 30.06 & 31.46 \\
\hline
\end{tabular}


Table 6 SSIM for noisy and filtered images. CR images generated at different exposure levels.

\begin{tabular}{ll|lllllll}
\hline \multicolumn{2}{c}{ Noise } & \multicolumn{7}{c}{ SSIM } \\
\hline Gaussian & Impulsive & Noisy & FPG & NDF & FPG-NDF & RLSF & SFRF & New filter \\
\hline \multicolumn{2}{l}{ CR images at $70 \mathrm{kV}$ with $0.4 \mathrm{mAs}$} \\
\hline $\begin{array}{l}l \\
\sigma=10\end{array} \quad p=0$ & 0.15 & 0.20 & 0.74 & 0.82 & 0.77 & 0.81 & 0.86 \\
$\sigma=0$ & $p=0.1$ & 0.11 & 0.86 & 0.28 & 0.85 & 0.74 & 0.87 & 0.95 \\
$\sigma=10$ & $p=0.1$ & 0.10 & 0.36 & 0.35 & 0.73 & 0.69 & 0.79 & 0.84 \\
\hline CR images at $80 \mathrm{kV}$ with $1 \mathrm{mAs}$ & & & & & & \\
\hline$\sigma=10$ & $p=0$ & 0.14 & 0.22 & 0.80 & 0.81 & 0.79 & 0.84 & 0.89 \\
$\sigma=0$ & $p=0.1$ & 0.12 & 0.89 & 0.29 & 0.82 & 0.74 & 0.88 & 0.98 \\
$\sigma=10$ & $p=0.1$ & 0.09 & 0.29 & 0.39 & 0.72 & 0.71 & 0.76 & 0.85 \\
\hline
\end{tabular}

Table 7 Computational time (seconds) on Multi-core and GPUs. Image mdb006.

\begin{tabular}{|c|c|c|c|c|c|c|c|c|}
\hline \multicolumn{2}{|c|}{ Noise } & \multicolumn{5}{|c|}{ Number of processors } & \multirow[t]{2}{*}{ GPU 1} & \multirow[t]{2}{*}{ GPU 2} \\
\hline Gaussian & Impulsive & 1 & 2 & 4 & 6 & 8 & & \\
\hline$\sigma=5$ & $p=0$ & 5.23 & 2.69 & 1.36 & 0.92 & 0.72 & 0.06429 & 0.00150 \\
\hline$\sigma=10$ & $p=0$ & 5.38 & 2.70 & 1.36 & 0.96 & 0.73 & 0.07452 & 0.00151 \\
\hline$\sigma=5$ & $p=0.05$ & 5.90 & 2.97 & 1.50 & 1.04 & 0.78 & 0.07870 & 0.00156 \\
\hline$\sigma=10$ & $p=0.1$ & 6.31 & 3.17 & 1.65 & 1.12 & 0.85 & 0.06848 & 0.00154 \\
\hline$\sigma=0$ & $p=0.05$ & 4.86 & 2.45 & 1.24 & 0.92 & 0.64 & 0.08130 & 0.00154 \\
\hline$\sigma=0$ & $p=0.1$ & 5.17 & 2.60 & 1.36 & 0.92 & 0.69 & 0.08294 & 0.00155 \\
\hline
\end{tabular}

Table 8 Computational time (seconds) on Multi-core and GPUs. Image RANDO phantom at $70 \mathrm{KV} 1 \mathrm{mAs}(3730 \times 3062$ pixels).

\begin{tabular}{|c|c|c|c|c|c|c|c|c|}
\hline \multicolumn{2}{|c|}{ Noise } & \multicolumn{5}{|c|}{ Number of processors } & \multirow[t]{2}{*}{ GPU 1} & \multirow[t]{2}{*}{ GPU 2} \\
\hline Gaussian & Impulsive & 1 & 2 & 4 & 6 & 8 & & \\
\hline$\sigma=5$ & $p=0$ & 121.03 & 61.51 & 29.60 & 18.38 & 12.73 & 0.18304 & 0.01715 \\
\hline$\sigma=10$ & $p=0$ & 124.51 & 61.74 & 29.60 & 19.31 & 12.96 & 0.18395 & 0.01727 \\
\hline$\sigma=5$ & $p=0.05$ & 136.60 & 68.01 & 32.85 & 21.17 & 14.12 & 0.19010 & 0.02014 \\
\hline$\sigma=10$ & $p=0.1$ & 146.12 & 72.66 & 36.34 & 23.03 & 15.75 & 0.14559 & 0.01752 \\
\hline$\sigma=0$ & $p=0.05$ & 112.43 & 55.93 & 26.81 & 18.38 & 10.87 & 0.19657 & 0.01626 \\
\hline$\sigma=0$ & $p=0.1$ & 119.63 & 59.42 & 29.60 & 18.38 & 12.03 & 0.20018 & 0.01656 \\
\hline
\end{tabular}

Table 9 Standard deviation. Computed radiography images at $70 \mathrm{KV}$.

\begin{tabular}{lcccc}
\hline & $0.4 \mathrm{mAs}$ & $0.6 \mathrm{mAs}$ & $0.8 \mathrm{mAs}$ & $1 \mathrm{mAs}$ \\
\hline Original & 29.2156 & 24.9404 & 24.5312 & 18.4855 \\
FPG-NDF & 21.9234 & 17.8742 & 17.4563 & 13.3467 \\
RLSF & 20.3504 & 16.8902 & 16.4156 & 12.1234 \\
SFRF & 20.1290 & 16.2784 & 16.0134 & 12.0212 \\
New Filter & 18.2351 & 15.7429 & 15.4429 & 11.9821 \\
\hline
\end{tabular}

2. Boncelet, C.: Image Noise Models, pp. 325-335. Academic Press, London (2000)

3. Camarena, J.G., Gregori, V., Morillas, S., Sapena, A.: Fast detection and removal of impulsive noise using peer groups and fuzzy metrics. J. Visual Commun. Image Represent. 19(1), 20-29 (2008)

4. Camarena, J.G., Gregori, V., Morillas, S., Sapena, A.: Some improvements for image filtering using peer group techniques. Image Vision Comput. 28(1), 188-201 (2010)

5. Camarena, J.G., Gregori, V., Morillas, S., Sapena, A.: Two-step fuzzy logic-based method for impulse noise detection in colour images. Pattern Recognit. Lett. 31(13), 1842-1849 (2010)
Table 10 Standard deviation. Computed radiography images at $80 \mathrm{KV}$.

\begin{tabular}{lcccc}
\hline & $0.4 \mathrm{mAs}$ & $0.6 \mathrm{mAs}$ & $0.8 \mathrm{mAs}$ & $1 \mathrm{mAs}$ \\
\hline Original & 24.0432 & 20.1867 & 19.9276 & 14.4075 \\
FPG-NDF & 17.1546 & 14.2312 & 14.1322 & 10.1053 \\
RLSF & 16.9574 & 14.0902 & 13.9145 & 10.0142 \\
SFRF & 16.5492 & 13.8682 & 13.8341 & 09.9872 \\
New Filter & 14.3351 & 12.4863 & 11.5278 & 09.3521
\end{tabular}

6. Camarena, J.G., Gregori, V., Morillas, S., Sapena, A.: A simple fuzzy method to remove mixed gaussian-impulsive noise from color images. IEEE Transactions on Fuzzy Systems 21(5), 971-978 (2013)

7. Chen, Y., Li, K., Yang, W., Xiao, G., Xie, X., Li, T.: Performance-aware model for sparse matrix-matrix multiplication on the sunway taihulight supercomputer. IEEE Transactions on Parallel and Distributed Systems (2018)

8. CUDA Home Page.: https://developer.nvidia.com/cudazone (2018). Accessed 12 December 2018

9. Dabov, K., Foi, A., Katkovnik, V., Egiazarian, K.: Image denoising by sparse 3 -d transform-domain collaborative 


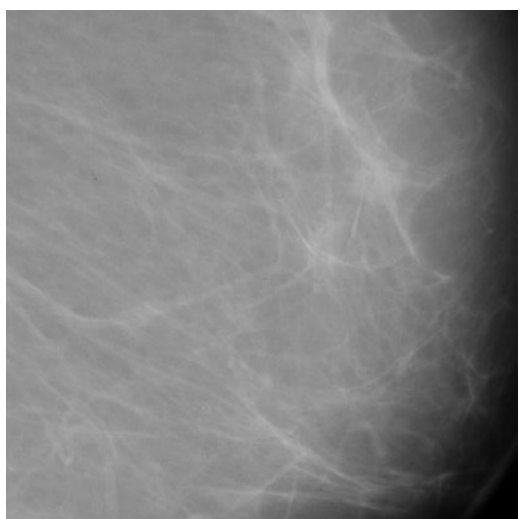

(a) Original mdb006

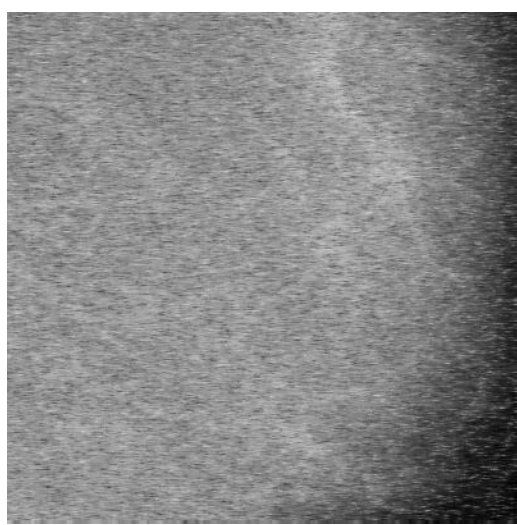

(d) NDF filter

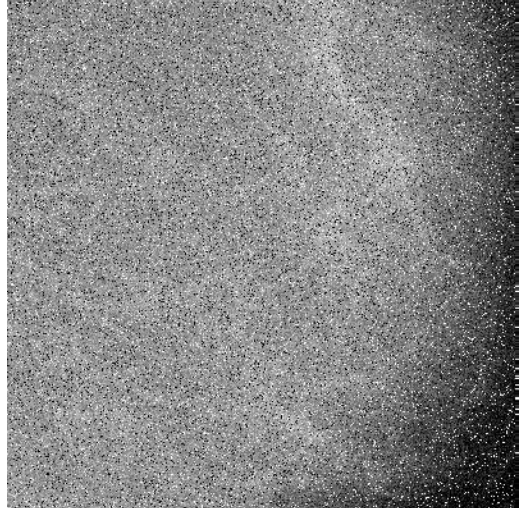

(b) Noisy $\sigma=10, p=0.1$

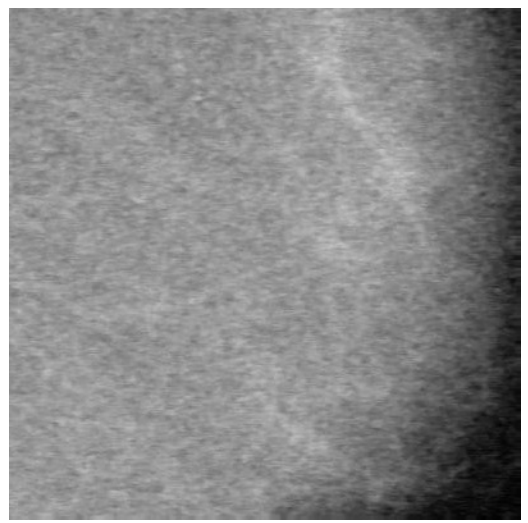

(e) FPG-NDF

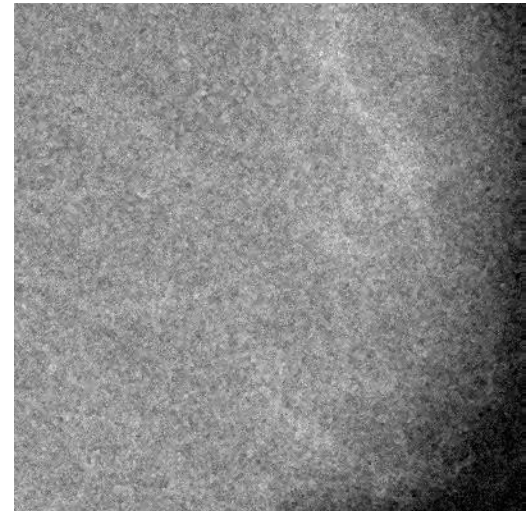

(c) FPG filter

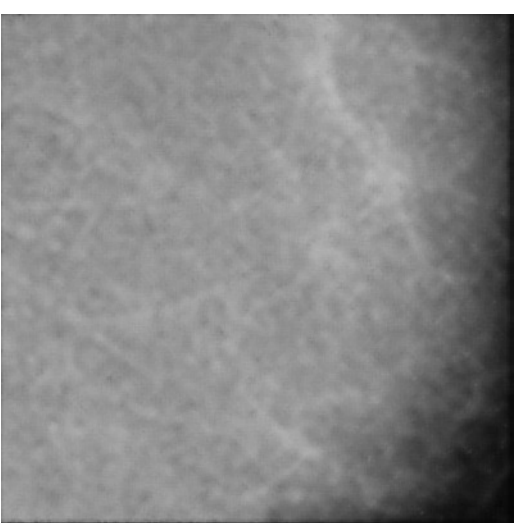

(f) RLSF

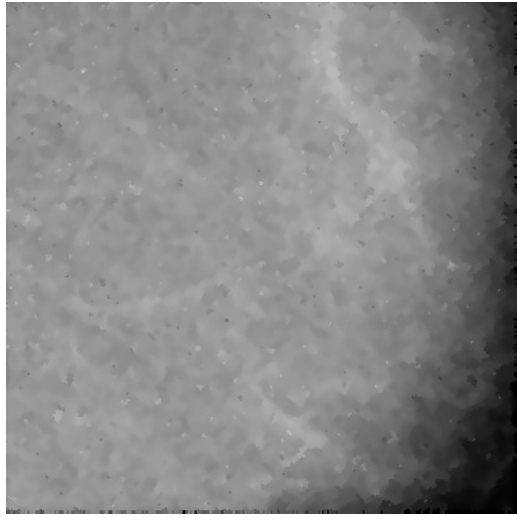

(g) SFRF

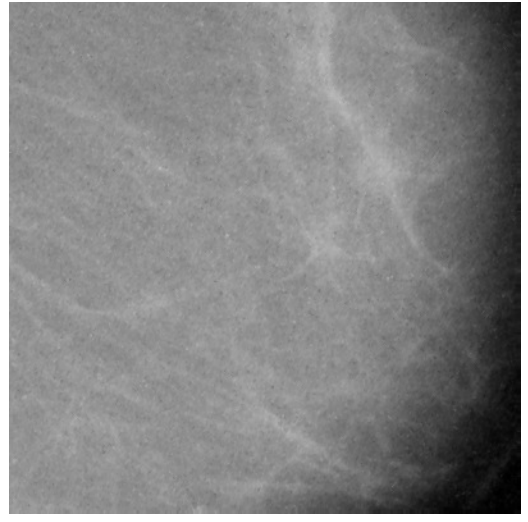

(h) New filter

Fig. 8 Filters output for visual comparison. Detail of mammogram mdb006 corrupted with $\sigma=10$ Gaussian noise, $p=0.1$ impulse.

filtering. Trans. Img. Proc. 16(8), 2080-2095 (2007).

10. Dabov, K., Foi, A., Katkovnik, V., Egiazarian, K.O.: Color image denoising via sparse $3 \mathrm{~d}$ collaborative filtering with grouping constraint in luminance-chrominance space. In: Proceedings of the International Conference on Image Processing, ICIP 2007, September 16-19, 2007, San Antonio, Texas, USA, pp. 313-316. IEEE (2007).

11. Dagum, L., Menon, R.: Openmp: an industry standard api for shared-memory programming. IEEE computational science and engineering 5(1), 46-55 (1998)
12. George, A., Veeramani, P.: On some results in fuzzy metric spaces. Fuzzy Sets Syst. 64(3), 395-399 (1994)

13. Gregori, V., Romaguera, S.: Characterizing completable fuzzy metric spaces. Fuzzy Sets Syst. 144(3), 411-420 (2004)

14. Kalra, M.K., Maher, M.M., Blake, M.A., Lucey, B.C., Karau, K., Toth, T.L., Avinash, G., Halpern, E.F., Saini, S.: Detection and characterization of lesions on lowradiation-dose abdominal ct images postprocessed with noise reduction filters. Radiology 232(3), 791-797 (2004) 


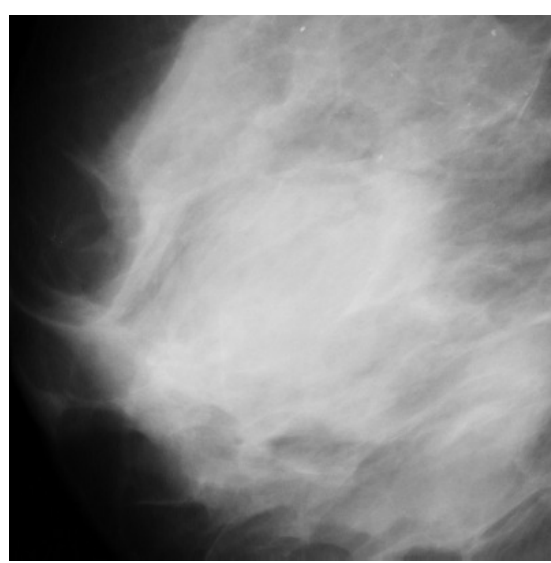

(a) Detail of original mammogram

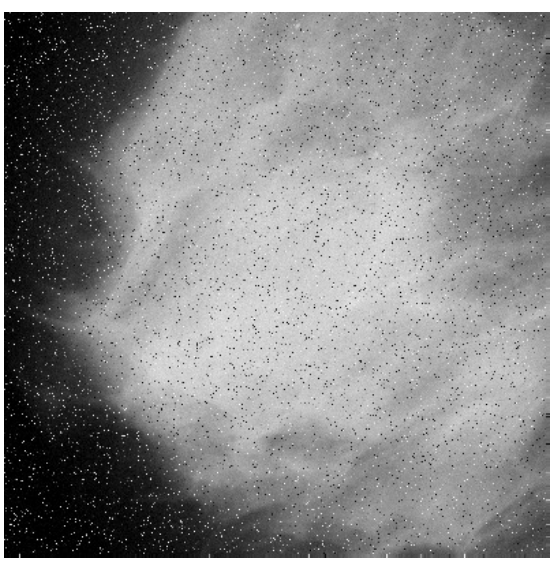

(b) Corrupted $\sigma=5$ Gaussian, $p=0.05$ impulsive

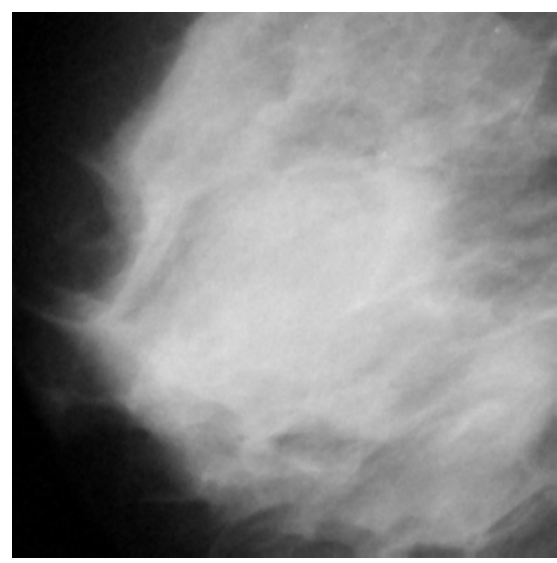

(c) Filtered mammogram

Fig. 9 Filter output for visual comparison. Detail of benign abnormality in mammogram mdb001

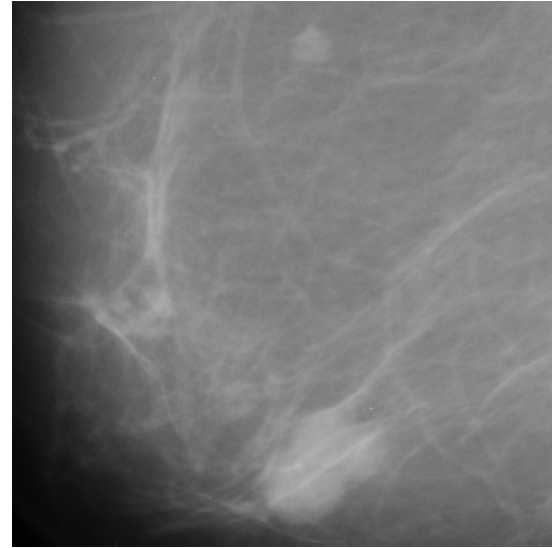

(a) Detail of original mammogram

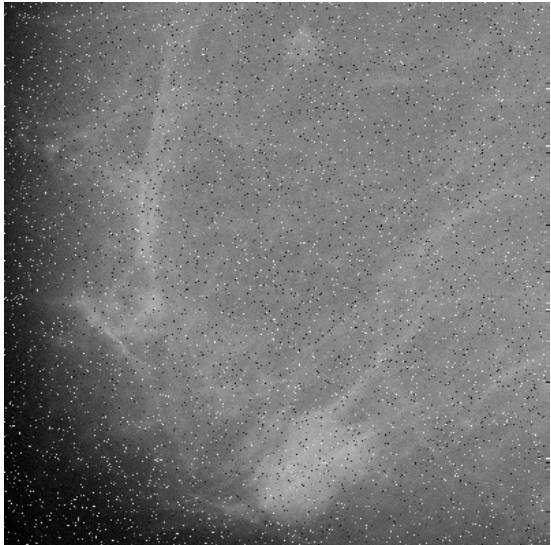

(b) Corrupted $\sigma=5$ Gaussian, $p=0.05$ impulsive

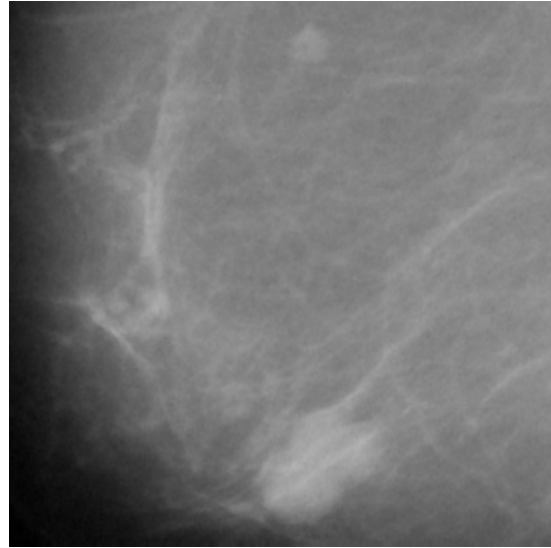

(c) Filtered mammogram

Fig. 10 Filter output for visual comparison. Detail of benign abnormality in mammogram mdb005

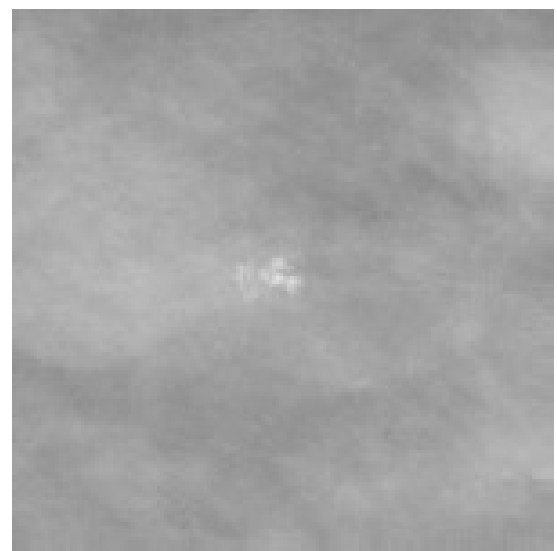

(a) Detail of original mammogram

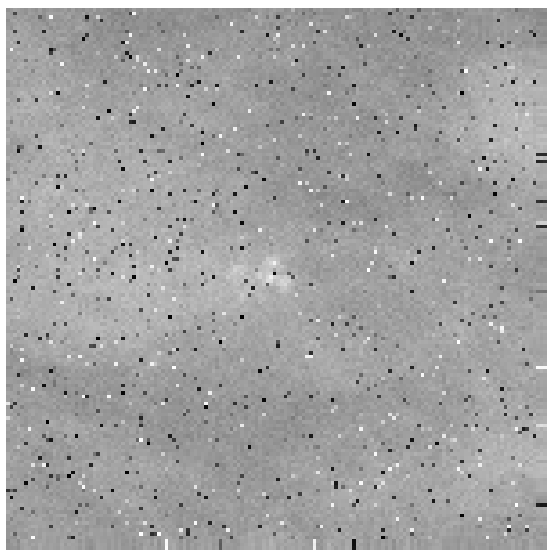

(b) Corrupted $\sigma=5$ Gaussian, $p=0.05$ impulsive

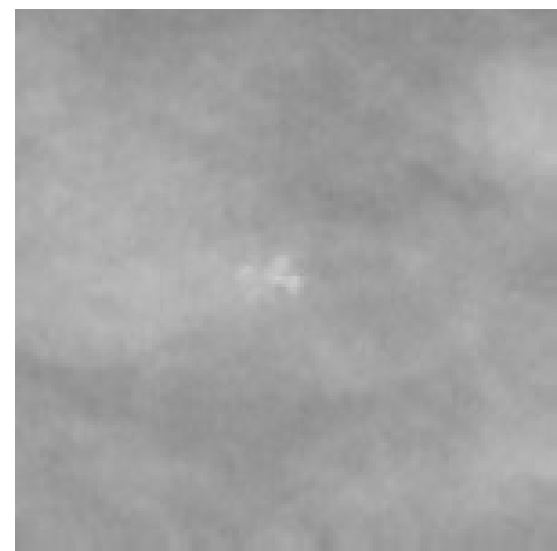

(c) Filtered mammogram

Fig. 11 Filter output for visual comparison. Detail of small region $(150 \times 150$ pixels $)$ containing microcalcifications in benign fatty mammogram mdb248 


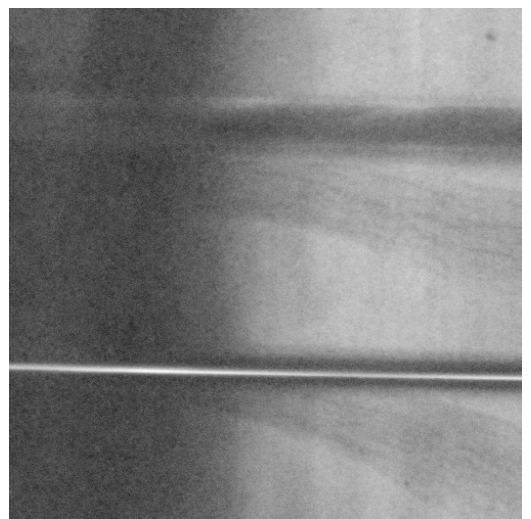

(a) Original CR image

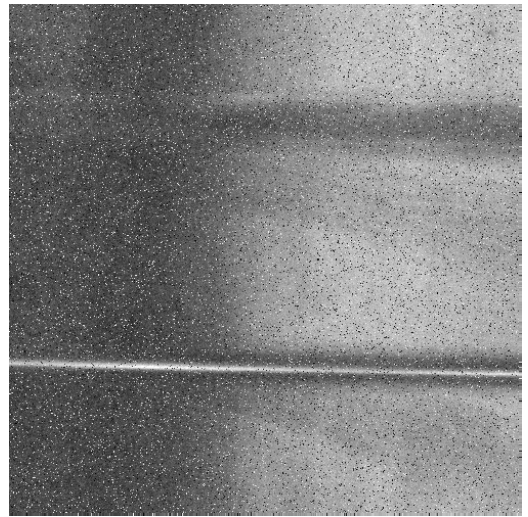

(b) Corrupted $\sigma=10$ Gaussian, $p=$ 0.1 impulsive

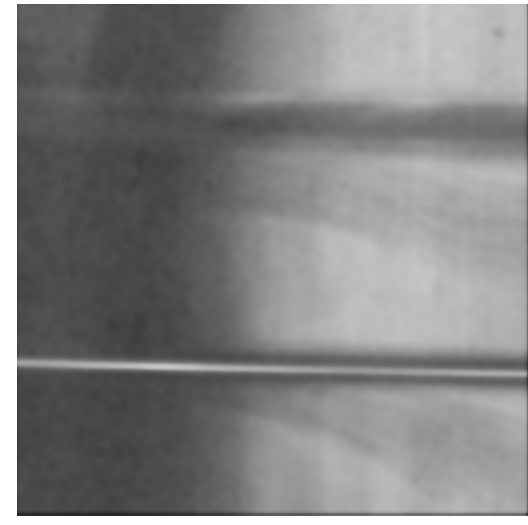

(c) Filtered RLSF

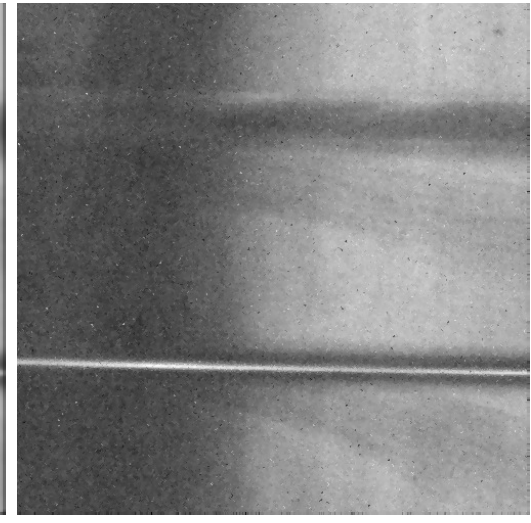

(d) Filtered SFRF

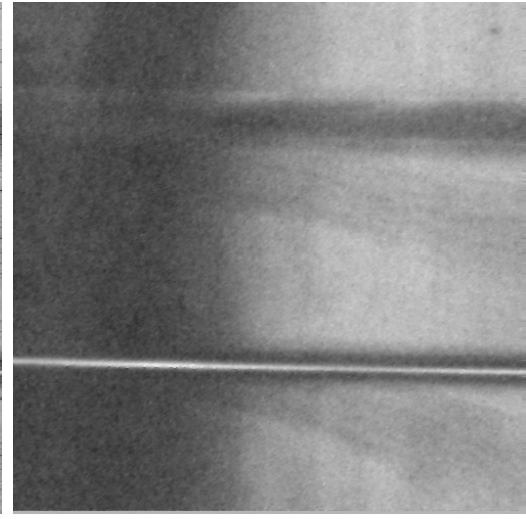

(e) Filtered New filter

Fig. $12 \mathrm{CR}$ images at $70 \mathrm{kV}$ with $0.4 \mathrm{mAs}$.

15. Kalra, M.K., Wittram, C., Maher, M.M., Sharma, A., Avinash, G.B., Karau, K., Toth, T.L., Halpern, E., Saini, S., Shepard, J.A.: Can noise reduction filters improve low-radiation-dose chest ct images pilot study. Radiology 228(1), 257-264 (2003)

16. Keeling, S.L.: Total variation based convex filters for medical imaging. Applied Mathematics and Computation 139(1), 101-119 (2003)

17. Kenney, C., Deng, Y., Manjunath, B.S., Hewer, G.: Peer group image enhancement. IEEE Trans. Image Process. 10(2), 326-334 (2001)

18. Li, K., Liu, C., Li, K., Zomaya, A.Y.: A framework of price bidding configurations for resource usage in cloud computing. IEEE Trans. Parallel Distrib. Syst. 27(8), 2168-2181 (2016).

19. Li, X.: On modeling interchannel dependency for color image denoising. Int. J. Imaging Syst. Technol. 17(3), 163-173 (2007)

20. Liu, C., Li, K., Xu, C., Li, K.: Strategy Configurations of Multiple Users Competition for Cloud Service Reservation. IEEE Trans. Parallel Distrib. Syst. 27(2), 508-520 (2016).

21. Melange, T., Nachtegael, M., Kerre, E.E.: Fuzzy random impulse noise removal from color image sequences. IEEE Trans. Image Process. 20(4), 959-970 (2011)

22. Morillas, S., Gregori, V., Hervás, A.: Fuzzy peer groups for reducing mixed gaussian-impulse noise from color images. IEEE Trans. Image Process. 18(7), 1452-1466 (2009)
23. Morillas, S., Gregori, V., Peris-Fajarnés, G.: Isolating impulsive noise pixels in color images by peer group techniques. Comput. Vision Image Understanding 110(1), $102-116(2008)$

24. Morillas, S., Gregori, V., Peris-Fajarnés, G., Sapena, A. Local self-adaptive fuzzy filter for impulsive noise removal in color images. Signal Process. 88(2), 390-398 (2008)

25. OpenMP ARB.: https://www.openmp.org (2018). Accessed 12 December 2018

26. Perona, P., Malik, J.: Scale-space and edge detection using anisotropic diffusion. IEEE Trans Pattern Anal Mach Intell 12(7), 629-639 (1990)

27. Plataniotis, K.N., Venetsanopoulos, A.N.: Color Image Processing And Applications. Springer-Verlag, New York, USA (2000)

28. Rudin, L.I., Osher, S., Fatemi, E.: Nonlinear total variation based noise removal algorithms. Physica D: Nonlinear Phenomena 60(1), 259 - 268 (1992)

29. Schulte, S., Huysmans, B., Pižurica, A., Kerre, E.E., Philips, W.: A new fuzzy-based wavelet shrinkage image denoising technique. In: Proceedings of the 8th international conference on Advanced Concepts For Intelligent Vision Systems, ACIVS'06, pp. 12-23. Springer-Verlag, Berlin, Heidelberg (2006)

30. Schulte, S., Morillas, S., Gregori, V., Kerre, E.E.: A new fuzzy color correlated impulse noise reduction method. IEEE Trans. Image Process. 16(10), 2565-2575 (2007)

31. Schulte, S., Nachtegael, M., Witte, V.D., der Weken, D.V., Kerre, E.E.: A fuzzy impulse noise detection and 


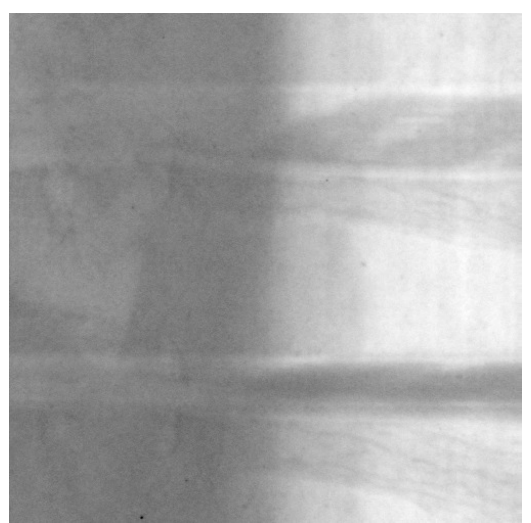

(a) Original CR image

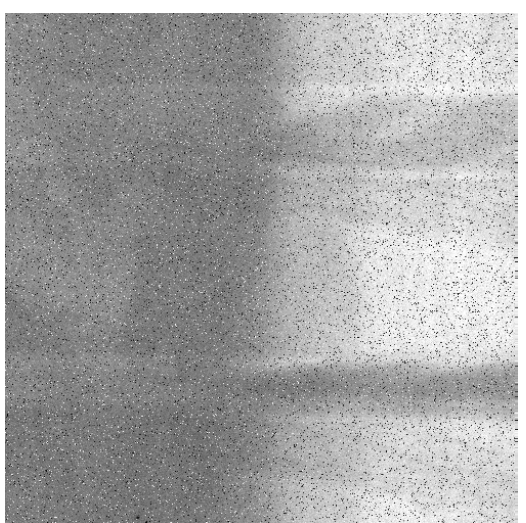

(b) Corrupted $\sigma=10$ Gaussian, $p=$ 0.1 impulsive

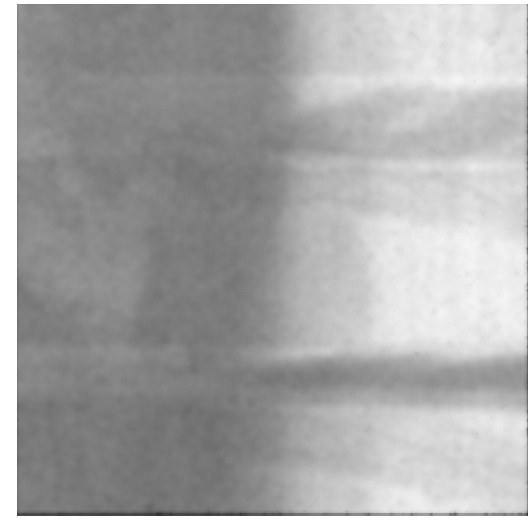

(c) Filtered RLSF

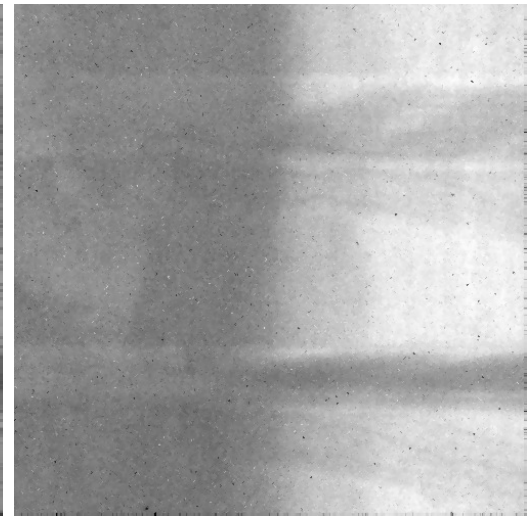

(d) Filtered SFRF

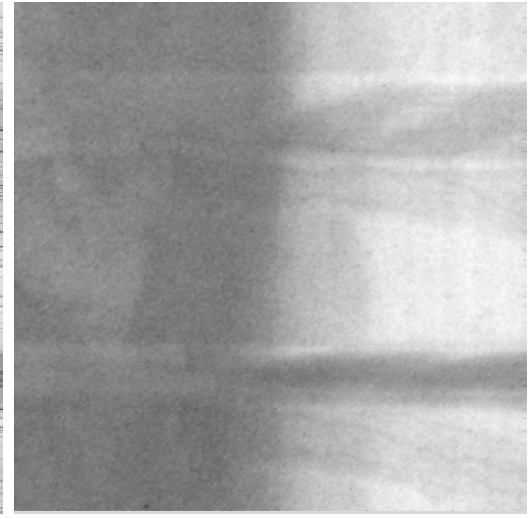

(e) Filtered New filter

Fig. $13 \mathrm{CR}$ images at $80 \mathrm{kV}$ with $1 \mathrm{mAs}$.

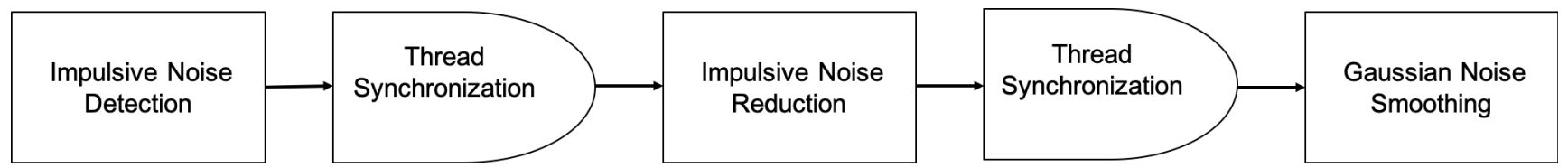

Fig. 14 Flowchart describing the kernel on the GPU

reduction method. IEEE Trans. Image Process. 15(5), 1153-1162 (2006)

32. Schulte, S., Witte, V.D., Nachtegael, M., der Weken, D.V., Kerre, E.E.: Fuzzy two-step filter for impulse noise reduction from color images. IEEE Trans. Image Process. 15(11), 3567-3578 (2006)

33. Schulte, S., Witte, V.D., Nachtegael, M., der Weken, D.V., Kerre, E.E.: Fuzzy random impulse noise reduction method. Fuzzy Sets Syst. 158(3), 270-283 (2007)

34. Smolka, B.: Peer group switching filter for impulse noise reduction in color images. Pattern Recognit. Lett. 31(6), 484-495 (2010)

35. Smolka, B., Chydzinski, A.: Fast detection and impulsive noise removal in color images. Real-Time Imaging 11(56), 389-402 (2005)

36. Smolka, B., Kusnik, D.: Robust local similarity filter for the reduction of mixed gaussian and impulsive noise in color digital images. Signal, Image and Video Processing $\mathbf{9}(1), 49-56(2015)$
37. Suckling, J., et al.: The mammographic image analysis society digital mammogram database. Exerpta Medica. International Congress Series 1069 pp. 375-378 (1994)

38. Tomasi, C., Manduchi, R.: Bilateral filtering for gray and color images. In: Proceedings of the Sixth International Conference on Computer Vision, ICCV '98, pp. 839-846. IEEE Computer Society, Washington, DC, USA (1998)

39. Toprak, A., Güler, I.: Impulse noise reduction in medical images with the use of switch mode fuzzy adaptive median filter. Digital Signal Process. 17(4), 711-723 (2007)

40. Wang, Y., Ren, W., Wang, H.: Anisotropic second and fourth order diffusion models based on convolutional virtual electric field for image denoising. Comput Math Appl 66(10), 1729 - 1742 (2013)

41. Wang, Z., Bovik, A.C., Sheikh, H.R., Simoncelli, E.P.: Image quality assessment: from error visibility to structural similarity. IEEE transactions on image processing 13(4), 600-612 (2004)

42. Wong, K.K., Fong, S., Wang, D.: Impact of advanced parallel or cloud computing technologies for image guided 
diagnosis and therapy. J. Xray. Sci. Technol. 25(2), 187192 (2017).

43. Xiao, G., Li, K., Li, K.: Reporting 1 most favorite objects in uncertain databases with probabilistic reverse top-k queries. In: Data Mining Workshop (ICDMW), 2015 IEEE International Conference on, pp. 1592-1599. IEEE (2015)

44. Xiao, G., Li, K., Li, K.: Reporting 1 most influential objects in uncertain databases based on probabilistic reverse top-k queries. Information Sciences 405, 207-226 (2017)

45. Xiao, G., Li, K., Li, K., Zhou, X.: Efficient top-(k, l) range query processing for uncertain data based on multicore architectures. Distributed and Parallel Databases 33(3), $381-413$ (2015) 